\title{
The Phosphate Binder Ferric Citrate Alters the Gut Microbiome in Rats with Chronic Kidney Disease ${ }^{\mathrm{Q}}$
}

\author{
Wei Ling Lau, Nosratola D. Vaziri, Ane C. F. Nunes, André M. Comeau, \\ Morgan G. I. Langille, Whitney England, Mahyar Khazaeli, Yasunori Suematsu, Joann Phan, \\ and Katrine Whiteson
}

\begin{abstract}
Division of Nephrology and Hypertension, Departments of Medicine (W.L.L., N.D.V., A.C.F.N., M.K.) and Molecular Biology and Biochemistry (W.E., J.P., K.W.), University of California, Irvine, California; Centre for Comparative Genomics and Evolutionary Bioinformatics Integrated Microbiome Resource, Department of Pharmacology, Dalhousie University, Halifax, Nova Scotia, Canada (A.M.C., M.G.I.L.); and Department of Cardiology, Fukuoka University, Fukuoka, Japan (Y.S.)
\end{abstract}

Received June 12, 2018; accepted September 28, 2018

\section{ABSTRACT}

In chronic kidney disease (CKD), the gut microbiome is altered and bacterial-derived uremic toxins promote systemic inflammation and cardiovascular disease. Ferric citrate complex is a dietary phosphate binder prescribed for patients with end-stage kidney disease to treat hyperphosphatemia and secondary hyperparathyroidism. Iron is an essential nutrient in both microbes and mammals. This study was undertaken to test the hypothesis that the large iron load administered with ferric citrate in CKD may significantly change the gut microbiome. Male Sprague-Dawley rats underwent $5 / 6$ nephrectomy to induce CKD. Normal control and CKD rats were randomized to regular chow or a $4 \%$ ferric citrate diet for 6 weeks. Fecal and cecal microbial DNA was analyzed via $16 \mathrm{~S}$ ribosomal RNA gene sequencing on the Illumina MiSeq system. CKD rats had lower abundances of Firmicutes and Lactobacillus compared with normal rats and had lower overall gut microbial diversity. CKD rats treated with ferric citrate had improved hemoglobin and creatinine clearance and amelioration of hyperphosphatemia and hypertension. Ferric citrate treatment increased bacterial diversity in CKD rats almost to levels observed in control rats. The tryptophanase-possessing families Verrucomicrobia, Clostridiaceae, and Enterobacteriaceae were increased by ferric citrate treatment. The uremic toxins indoxyl sulfate and $p$-cresyl sulfate were not increased with ferric citrate treatment. Verrucomicrobia was largely represented by Akkermansia muciniphila, which has important roles in mucin degradation and gut barrier integrity. In summary, ferric citrate therapy in CKD rats was associated with significant changes in the gut microbiome and beneficial kidney and blood pressure parameters.

\section{Introduction}

Systemic inflammation is invariably present in humans and animals with chronic kidney disease (CKD) and is marked by activation of circulating leukocytes and elevation of plasma proinflammatory cytokines and chemokines (Yoon et al., 2007; Kato et al., 2008; Heine et al., 2012). CKD-associated systemic inflammation and oxidative stress play a central role in the pathogenesis of numerous complications of CKD, including accelerated cardiovascular disease, frailty, and anemia, among others (Himmelfarb et al., 2002; Vaziri, 2004; Cachofeiro et al., 2008). There are profound changes in

This work was supported by an unrestricted research grant from Keryx Biopharmaceuticals Inc. W.L.L. has received funding from the American Heart Association, Hub Therapeutics, Sanofi, and the Division of Nephrology at University of California, Irvine. N.D.V. has received funding from Reata, Keryx Biopharmaceuticals, and Novartis. A.C.F.N. received support from a Brazil Science Without Borders grant. Y.S. received support from a Sumitomo Life Welfare and Culture Foundation and Overseas Research Scholarship and a Fukuoka University School of Medicine Alumni Overseas Research Scholarship.

https://doi.org/10.1124/jpet.118.251389.

S This article has supplemental material available at jpet.aspetjournals.org. the structure and function of the gut microbiome and disruption of the gut epithelial barrier in CKD (Vaziri et al., 2012, 2013a,b). Increased permeability of the intestinal epithelium in CKD is evidenced by the appearance of orally administered high molecular weight polyethylene glycols in the urine (Magnusson et al., 1990, 1991). Several studies have demonstrated the role of gut microbial-derived uremic toxins such as indoxyl sulfate, $p$-cresyl sulfate, and trimethylamine $\mathrm{N}$-oxide in the pathogenesis of CKDassociated systemic inflammation (Liabeuf et al., 2010; Aronov et al., 2011; Tang et al., 2015; Stubbs et al., 2016). Endotoxin, derived from the cell wall of Gram-negative bacteria, is measurable in the blood of patients with CKD and increases with severity of CKD stage, being most elevated in chronic dialysis patients (Szeto et al., 2008; McIntyre et al., 2011).

Several factors contribute to the gut microbial dysbiosis in patients with advanced CKD. First, accumulation of urea in the body fluids and its diffusion in the gastrointestinal tract leads to the expansion of urease-possessing bacteria (Wong et al., 2014). Hydrolysis of urea by these microbial species

ABBREVIATIONS: ANOVA, analysis of variance; CKD, chronic kidney disease; FC, ferric citrate group; NL, normal control group; NMDS, nonmetric multidimensional scaling; OTU, operational taxonomic unit; PERMANOVA, permutational multivariate analysis of variance. 
results in the formation of ammonia and caustic ammonium hydroxide $\left[\mathrm{CO}\left(\mathrm{NH}_{2}\right)_{2}+\mathrm{H}_{2} \mathrm{O} \rightarrow \mathrm{CO}_{2}+2 \mathrm{NH}_{3} ; \mathrm{NH}_{3}+\mathrm{H}_{2} \mathrm{O} \rightarrow\right.$ $\mathrm{NH}_{4} \mathrm{OH}$ ], which degrade the epithelial tight junction (Vaziri et al., 2013c), thereby facilitating translocation of endotoxin and microbial fragments into the systemic circulation (Wang et al., 2012; Lau et al., 2015; Vaziri et al., 2016). Second, dietary restrictions to limit hyperkalemia and hyperphosphatemia in CKD correlate with a diet low in potassium-rich (fruits and vegetables) and phosphate-rich (cheese and yogurt) products. These dietary restrictions result in the unintended reduction of indigestible complex carbohydrates, a nutrient source for gut microbial flora, from which they generate short-chain fatty acids. Short-chain fatty acids in turn are major nutrients for colonocytes and are essential for the integrity of the colonic epithelium. In addition, the reduction of short-chain fatty acid production and formation of ammonium hydroxide leads to a rise in the $\mathrm{pH}$ of the colonic milieu, which further impacts the gut microbial community (Sirich et al., 2014; Kieffer et al., 2016). Furthermore, the limited intake of cheese and yogurt reduces exposure to useful symbiotic bacteria. Third, pharmaceutical interventions including commonly prescribed phosphate or potassium binders significantly alter the biochemical milieu of the gut and can potentially impact the gut microbiome. However, to our knowledge, the effect of these compounds on the gut microbiome in CKD has not been previously investigated.

Ferric citrate is a calcium-free iron compound used as a dietary phosphorus binder to manage hyperphosphatemia in patients with end-stage kidney disease (Yokoyama et al., 2012, 2014; Lee et al., 2015; Lewis et al., 2015), administered in doses that provide $2-4 \mathrm{~g}$ elemental iron per day. Ferric citrate has been shown to ameliorate secondary hyperparathyroidism and vascular calcification in CKD (Iida et al., 2013; Block et al., 2015). Ferric citrate has additional benefits, in that it improves iron deficiency anemia by restoring iron stores. In a phase III randomized controlled trial of ferric citrate therapy in nondialysis patients with $\mathrm{CKD}$, mean transferrin saturation and ferritin were increased by $18.4 \%$ and $170 \mathrm{ng} / \mathrm{ml}$, respectively, after 16 weeks of therapy (Fishbane et al., 2017).

Iron is an essential nutrient in both microbes and mammals. Microbes acquire iron by producing siderophores, which are small molecules that chelate and internalize iron. Siderophores play a major role in microbial physiology and virulence, and they can modulate interbacterial competition and host cellular pathways (Holden and Bachman, 2015; Wilson et al., 2016). However, it is unknown whether gut microbes would be affected by ferric citrate therapy. Studies in non-CKD rodents have demonstrated varying effects of ferrous sulfate supplementation on the gut microbiome, including a decrease in the proportion of strict anaerobes (Benoni et al., 1993; Tompkins et al., 2001; Alexeev et al., 2017). Studies in CKD models to examine the impact of iron supplementation on the gut microbiome are lacking. Given the critical role of iron in microbial growth and virulence, this study was undertaken to test the hypothesis that the large iron load administered with ferric citrate in CKD may result in significant changes in the gut microbiome. To this end, the fecal microbiome was characterized in both normal and CKD rats treated with or without ferric citrate in their chow for 6 weeks.

\section{Materials and Methods}

Animals. All experiments were approved by the University of California, Irvine Institutional Committee for the Use and Care of Experimental Animals. Eight-week-old male Sprague-Dawley rats were purchased from Charles River Laboratories (Raleigh, NC). They were housed in a climate-controlled vivarium with 12-hour day/night cycles and were provided access to food and water ad libitum. The CKD groups were subjected to $5 / 6$ nephrectomy by removing the upper and lower thirds of the decapsulated left kidney, followed by right nephrectomy 7 days later as described previously (Vaziri et al., 2007). The normal control group (NL) underwent a sham operation. General anesthesia was induced with $5 \%$ inhaled isoflurane (Piramal Critical Care, Bethlehem, PA) and maintained at 2\%-4\% isoflurane during surgery. For pain relief, rats were given $0.05 \mathrm{mg} / \mathrm{kg}$ Buprenex (Reckitt Benckiser Pharmaceutical Inc., Richmond, VA). The NL and CKD groups were randomly assigned to a regular diet or a diet containing $4 \%$ ferric citrate for 6 weeks (denoted as the NL+FC and $\mathrm{CKD}+\mathrm{FC}$ groups, respectively). The animals were then placed in metabolic cages for a 24-hour urine collection. Systolic blood pressure was measured by tail plethysmography as described previously (Vaziri et al., 2002). Animals were euthanized by cardiac exsanguination under isoflurane anesthesia and colons were resected. Cecal and colon stool contents were collected and processed for determination of microbial community composition as described below.

Blood and Urine Biochemistries. Plasma phosphorus, calcium, iron, blood urea nitrogen, and urine creatinine were determined using QuantiChrom Assay Kits from BioAssay Systems (Hayward, CA). Plasma creatinine was measured using capillary electrophoresis at the O'Brien Kidney Research Core Center (University of Texas Southwestern, Dallas, TX). Blood hemoglobin was determined using the AimStrip Hb meter (Ermarine Laboratories Inc., San Antonio, TX).

Mass Spectrometry for Indoxyl Sulfate and $p$-Cresyl Sulfate. Analysis was done at the Mass Spectrometry Facility at the University of California, Irvine Chemistry Department (Irvine, CA). We used a modified protocol based on previously described methods (Shu et al., 2016; Kanemitsu et al., 2017). A 0.1-ml aliquot of plasma was treated with $200 \mu \mathrm{l}$ acetonitrile for protein precipitation containing $2 \mu \mathrm{g} / \mathrm{ml}$ hydrochlorothiazide. Hydrochlorothiazide was used as the internal standard. The mixture was vortexed and homogenized for 10 minutes in a water bath sonicator then centrifuged at $16,400 \mathrm{~g}$ for 15 minutes at $4^{\circ} \mathrm{C}$. The supernatant was collected into a 2 -ml microcentrifuge tube and evaporated to dryness at $60^{\circ} \mathrm{C}$. The dried extract was reconstituted with $100 \mu \mathrm{l} 25 \%$ acetonitrile.

Indoxyl sulfate, $p$-cresyl sulfate, and hydrochlorothiazide reference standards were purchased from Fisher Scientific (cat. nos. 501151154, AAA1707901, and 5001437615; Rockford, IL). A stock $2 \mathrm{mg} / \mathrm{ml}$ solution was prepared with water and dilutions were prepared with $25 \%$ acetonitrile with $4 \mu \mathrm{g} / \mathrm{ml}$ hydrochlorothiazide (internal standard) to generate a standard curve ranging from 1 to $2000 \mathrm{ng} / \mathrm{ml}$. Standards and prepared samples were injected $(10 \mu \mathrm{l})$ into the high-performance liquid chromatography-tandem mass spectrometry instrument, a Waters Quattro Premier XE (Waters, Milford, MA) equipped with a ultra-performance liquid chromatography system. The ultraperformance liquid chromatography system has a BEH C18 column, which allows rapid sample throughput. Mobile phase A was water with $5 \mathrm{mM}$ ammonium formate, and mobile phase B was 95\% methanol with $5 \mathrm{mM}$ ammonium formate. Analysis was performed in negative ionization mode by using multiple reaction monitoring tandem mass spectrometry with standard calibration. The transition $(\mathrm{m} / z)$ values were as follows: indoxyl sulfate, $211.97>80.36 ; p$-cresyl sulfate, $186.94>107.30$; and hydrochlorothiazide, $296.96>270.08$.

Microbial DNA Extraction and 16S Ribosomal RNA Gene Amplicon Sequencing. Cecal and fecal samples stored at $-80^{\circ} \mathrm{C}$ were sent to the Centre for Comparative Genomics and Evolutionary Bioinformatics Integrated Microbiome Resource at Dalhousie University (Halifax, NS, Canada) for extraction, library preparation, and 
sequencing of the 16S V6-V8 region on an Illumina MiSeq system (San Diego, CA). Microbial DNA was extracted with a Mo Bio PowerFecal extraction kit (QIAGEN, Germantown, MD), and amplification and sequencing was done as previously described (Comeau et al., 2017) (also see http://cgeb-imr.ca/protocols.html).

Sequence Analysis. A total of 32,000 sequences per sample were retained following quality control filtering and rarefaction, and these remaining sequences were clustered into $97 \%$ operational taxonomic units (OTUs) using open-reference clustering. All steps were conducted with Microbiome Helper, as described previously (Rossi et al., 2014). The resulting OTU table was then used for further analysis within the Vegan package in $\mathrm{R}$ software (R Foundation for Statistical Computing, Vienna, Austria) (Oksanen et al., 2007). $\alpha$ - and $\beta$-diversity metrics were calculated, and data were also analyzed using the STAMP software package (Parks et al., 2014). $\alpha$-diversity metrics assess both the richness (number of taxa) and evenness (distribution of species abundances). We included three metrics of $\alpha$ diversity that each combine richness and evenness: Shannon, Simpson (this metric gives more weight to dominant species), and Fisher analyses as implemented in the Vegan package in R (Oksanen et al., 2007).

Statistical Analysis. Data were screened for outliers using the Grubbs' test (extreme studentized deviate method, http://graphpad. com/quickcalcs/grubbs1/). Group data were analyzed using one-way analysis of variance (ANOVA) with the post hoc Tukey test, and $P<$ 0.05 was considered significant. A nonparametric statistical test was applied to verify the clustering of microbial diversity. Permutational multivariate analysis of variance (PERMANOVA) was applied to determine which factors (i.e., being in the CKD group or receiving ferric citrate treatment) explained the most variation in microbial community composition.

\section{Results}

General Data. Data are shown in Table 1 for the four study groups: NL $(n=5), \mathrm{NL}+\mathrm{FC}(n=6), \mathrm{CKD}(n=4)$, and $\mathrm{CKD}+\mathrm{FC}(n=6)$. Compared with the CKD rats consuming regular chow, the CKD rats consuming a diet supplemented with $4 \%$ ferric citrate showed a significant increase in plasma iron and blood hemoglobin concentration, a modest decline in arterial pressure and plasma creatinine concentration, and a modest rise in creatinine clearance, but no significant change in blood urea concentration. Plasma phosphorus levels were decreased as expected, as ferric citrate is a dietary phosphate binder.

Plasma levels of the gut-derived uremic toxins indoxyl sulfate and $p$-cresyl sulfate were increased in CKD animals compared with NL sham controls, but they were not significantly different between the CKD groups. Indoxyl sulfate was
$594 \pm 69$ versus $2253 \pm 328$ versus $2235 \pm 226 \mathrm{ng} / \mathrm{ml}$ in the $\mathrm{NL}$ versus $\mathrm{CKD}$ versus $\mathrm{CKD}+\mathrm{FC}$ groups (mean \pm S.E.M., ANOVA $P<0.01$ with post hoc Tukey $P<0.05$ between NL vs. $\mathrm{CKD}$ and $\mathrm{NL}$ vs. $\mathrm{CKD}+\mathrm{FC}$ ), respectively. The $p$-cresyl sulfate levels were $113 \pm 51$ versus $450 \pm 150$ versus $491 \pm 100 \mathrm{ng} / \mathrm{ml}$ in the NL versus CKD versus CKD $+\mathrm{FC}$ groups (ANOVA $P=$ 0.05 and nonsignificant on pairwise comparisons), respectively.

Gut Microbial Communities in CKD Rats Are Less Diverse. A total of $42 \mathrm{fecal}$ and cecal samples from the four to six animals in each of the four treatment groups were processed for microbiome analysis. DNA was extracted and then the V6-V8 region of the 16S ribosomal RNA gene was amplified and sequenced as described in the Materials and Methods. Distributions of taxa per phyla and the highest level of resolution achievable are shown in Fig. 1; the phyla Firmicutes and Bacteroides comprised the majority of all communities. CKD rats (treated and untreated) had higher levels of Bacteroidetes and fewer Firmicutes compared with control rats $(P<0.01$ across groups), which had an inverse Bacteroidetes/Firmicutes ratio (Fig. 1A; Supplemental Fig. 1). Figure 1B shows the distribution of taxa identified at the most resolved level of taxonomy, usually genus but sometimes order or family. For example, the second most abundant OTU was an uncultured Bacteroides (f_S24-7 as shown in Fig. 1B), a family that is often found at high levels in the guts of homeothermic mammals (Ormerod et al., 2016).

Rarefaction curves, displaying the number of bacterial types as a function of the number of sequence reads that were sampled, are shown in Fig. 2 and Supplemental Fig. 2 and suggest that the CKD rats, and especially the untreated control CKD rats, had lower and more variable gut microbial diversity. The diversity metrics displayed in Fig. 3 also demonstrate the variability of the CKD rats, with some samples displaying significantly less diversity. Analysis of fecal samples showed a mean Fisher's $\alpha$ of 177 versus 174 in the NL versus NL+FC groups and 114 versus 141 in the CKD versus $\mathrm{CKD}+\mathrm{FC}$ groups $(P<0.05$ across groups with significant differences between NL vs. CKD and NL+FC vs. CKD). $\alpha$ diversity was decreased $36 \%$ in the CKD group compared with NL animals and was decreased less in the $\mathrm{CKD}+\mathrm{FC}$ group (20\% less diversity compared with NL). Cecal samples were not significantly different across groups $(P=$ 0.3 ) although there was a similar trend for decreased diversity in the CKD groups (Fisher's $\alpha$ decreased $20 \%$ and $10 \%$ in the $\mathrm{CKD}$ and $\mathrm{CKD}+\mathrm{FC}$ groups, respectively, compared with NL animals).

TABLE 1

Body weight, tail blood pressure, and plasma and urine data in the four study groups Data are mean \pm S.E.M. $(n=4-6$ per group $)$.

\begin{tabular}{|c|c|c|c|c|}
\hline \multirow{2}{*}{ Variable } & \multicolumn{2}{|c|}{ NL Rats } & \multicolumn{2}{|c|}{ 5/6 Nephrectomy CKD Rats } \\
\hline & NL & $\mathrm{NL}+\mathrm{FC}$ & CKD & $\mathrm{CKD}+\mathrm{FC}$ \\
\hline Systolic blood pressure (mm Hg) & $108.7 \pm 3.1$ & $111.7 \pm 1.1$ & $149.0 \pm 3.4^{*}, \dagger$ & $112.8 \pm 4.8 \div$ \\
\hline Body weight, week 6 (g) & $408.3 \pm 12.8$ & $387.8 \pm 8.4$ & $290.8 \pm 18.3^{*}, \dagger$ & $357.8 \pm 15.5^{*}, 末$ \\
\hline Plasma phosphorus (mg/dl) & $8.5 \pm 0.2$ & $8.7 \pm 0.2$ & $12.2 \pm 0.2^{*}, \dagger$ & $8.7 \pm 0.6 \ddagger$ \\
\hline Plasma calcium $(\mathrm{mg} / \mathrm{dl})$ & $9.8 \pm 0.7$ & $10.4 \pm 0.4$ & $9.0 \pm 0.5$ & $9.8 \pm 0.3$ \\
\hline Plasma total iron $(\mu \mathrm{g} / \mathrm{dl})$ & $218.4 \pm 20.4$ & $285.0 \pm 19.1$ & $167.1 \pm 7.5 \dagger$ & $196.2 \pm 21.8 \dagger$ \\
\hline Hemoglobin (g/dl) & $14.9 \pm 0.1$ & $14.8 \pm 0.1$ & $8.6 \pm 0.8^{*}, \dagger$ & $12.4 \pm 0.4^{*}, \dagger, \dagger$ \\
\hline Blood urea nitrogen $(\mathrm{mg} / \mathrm{dl})$ & $20.1 \pm 0.5$ & $20.4 \pm 0.3$ & $160.9 \pm 25.9^{*}, \dagger$ & $93.6 \pm 26.2 \dagger$ \\
\hline Plasma creatinine $(\mathrm{mg} / \mathrm{dl})$ & $0.2 \pm 0.01$ & $0.2 \pm 0.03$ & $2.6 \pm 0.2^{*}, \dagger$ & $1.1 \pm 0.2^{*}, \dagger, \ddagger$ \\
\hline Creatinine clearance $(\mathrm{ml} / \mathrm{min} * \mathrm{~kg})$ & $10.3 \pm 2.0$ & $12.1 \pm 1.1$ & $0.8 \pm 0.1^{*}, \dagger$ & $2.5 \pm 0.3^{*}, \dagger$ \\
\hline
\end{tabular}

${ }^{*} P<0.05$ vs. NL; ${ }^{\dagger} P<0.05$ vs. $\mathrm{NL}+\mathrm{FC} ;{ }^{*} P<0.05$ vs. CKD. 
A
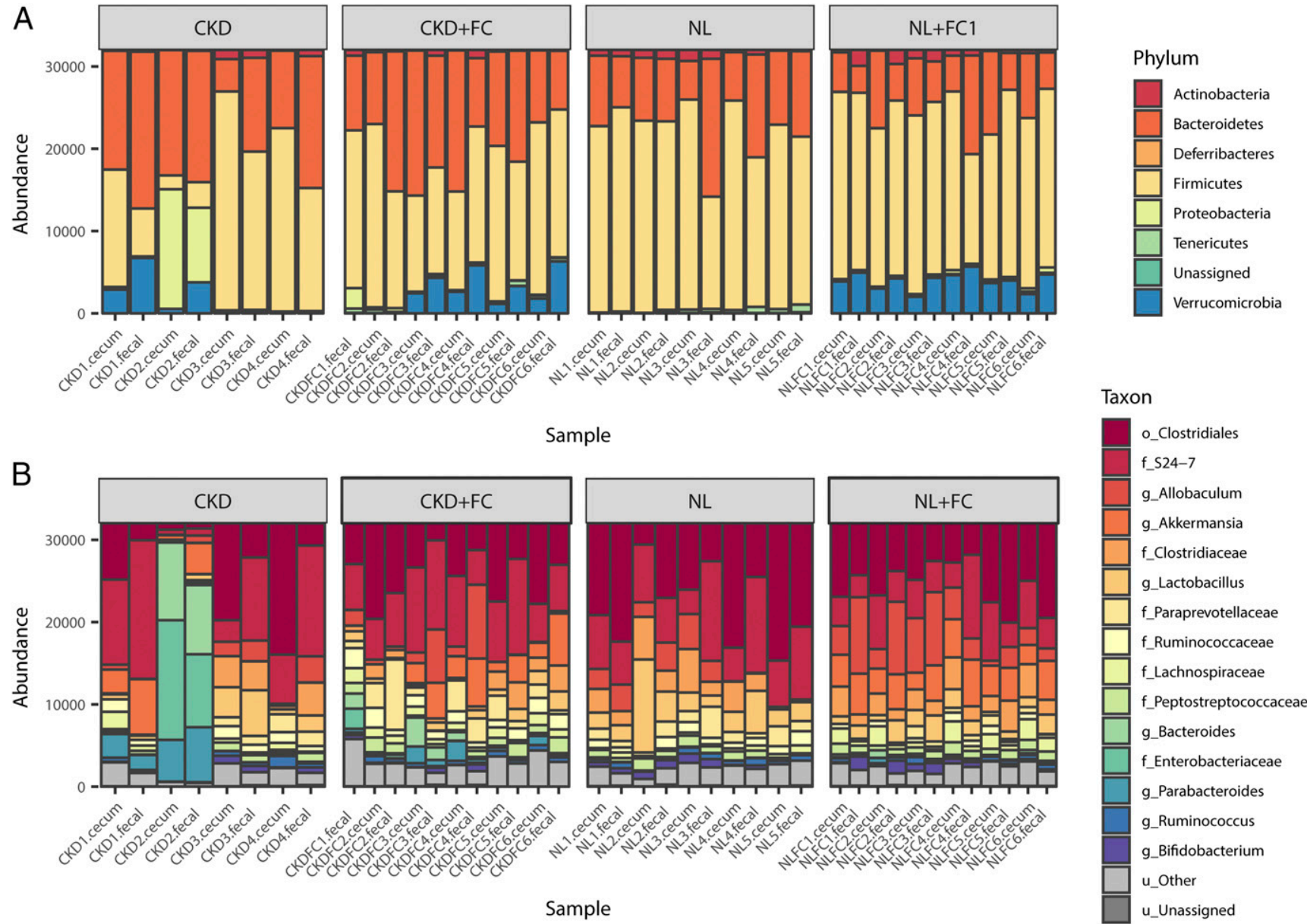

Fig. 1. (A and B) Relative abundance of bacterial taxa at the phylum level (A) and the highest level of resolution achievable (B) from 16S ribosomal RNA gene sequencing and 97\% OTU clusters. Fecal and cecal samples were from untreated and FC-treated NL and CKD rats. The second most abundant taxon at the genus level is an uncultured Bacteroidetes ( $f_{-}$S24-7) that is often found in the gut communities of homeothermic mammals. f, family; $g$, genus; o, order; $\mathrm{u}$, unassigned.

A Bray-Curtis distance matrix is displayed as a nonmetric multidimensional scaling (NMDS) ordination plot in Fig. 4. In these types of plots, samples with more similar microbial community composition are located closer to each other on the plot. This plot suggests that the microbial communities of individual NL rats were more similar to each other in composition than those from the CKD rats, as shown by the greater spread of the CKD-derived samples in the NMDS plot (Fig. 4).

Ferric Citrate Treatment Affects Gut Microbial Composition in Both Normal and CKD Rats. Ferric citrate treatment in both NL and CKD animals shifted the samples toward the origin of the vertical axis, NMDS2 (Fig. 4), so that they were clustered together more closely, with less widespread individual variability. Treatment with ferric citrate increased diversity in CKD rats, almost to the levels observed in NL rats (Fig. 3), and reduced abundances of Firmicutes in both normal and CKD rats (Fig. 1A; Supplemental Fig. 1). The Verrucomicrobia, which were largely Akkermansia muciniphila, had elevated abundances in the nontreated CKD rats that increased further with ferric citrate treatment in both the NL and CKD rat groups (Fig. 5). A. muciniphila increased from near zero to $12 \%$ of the community in the treated NL rats (Fig. 5). Similarly, other tryptophanase-possessing families, the Clostridiaceae and Enterobacteriaceae, were increased by ferric citrate treatment in both NL and CKD animals (data not shown).

A nonparametric statistical test was applied to verify the groupings that can be seen in Fig. 4. PERMANOVA is a multivariate ANOVA that tests the null hypothesis that there are no differences in microbial community composition between the health status or treatment groups, and it was applied to determine which variables explained the most

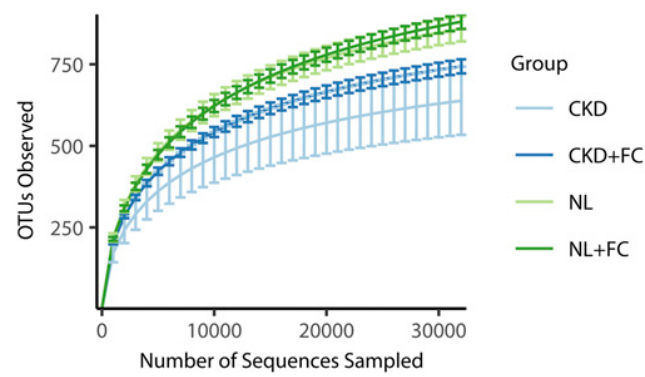

Fig. 2. Rarefaction curve showing the number of $97 \%$ identity bacterial OTUs from fecal samples in the four study groups with or without ferric citrate therapy. The $x$-axis shows the number of sequences sampled to calculate the corresponding "OTUs observed" value for the $y$-axis. 

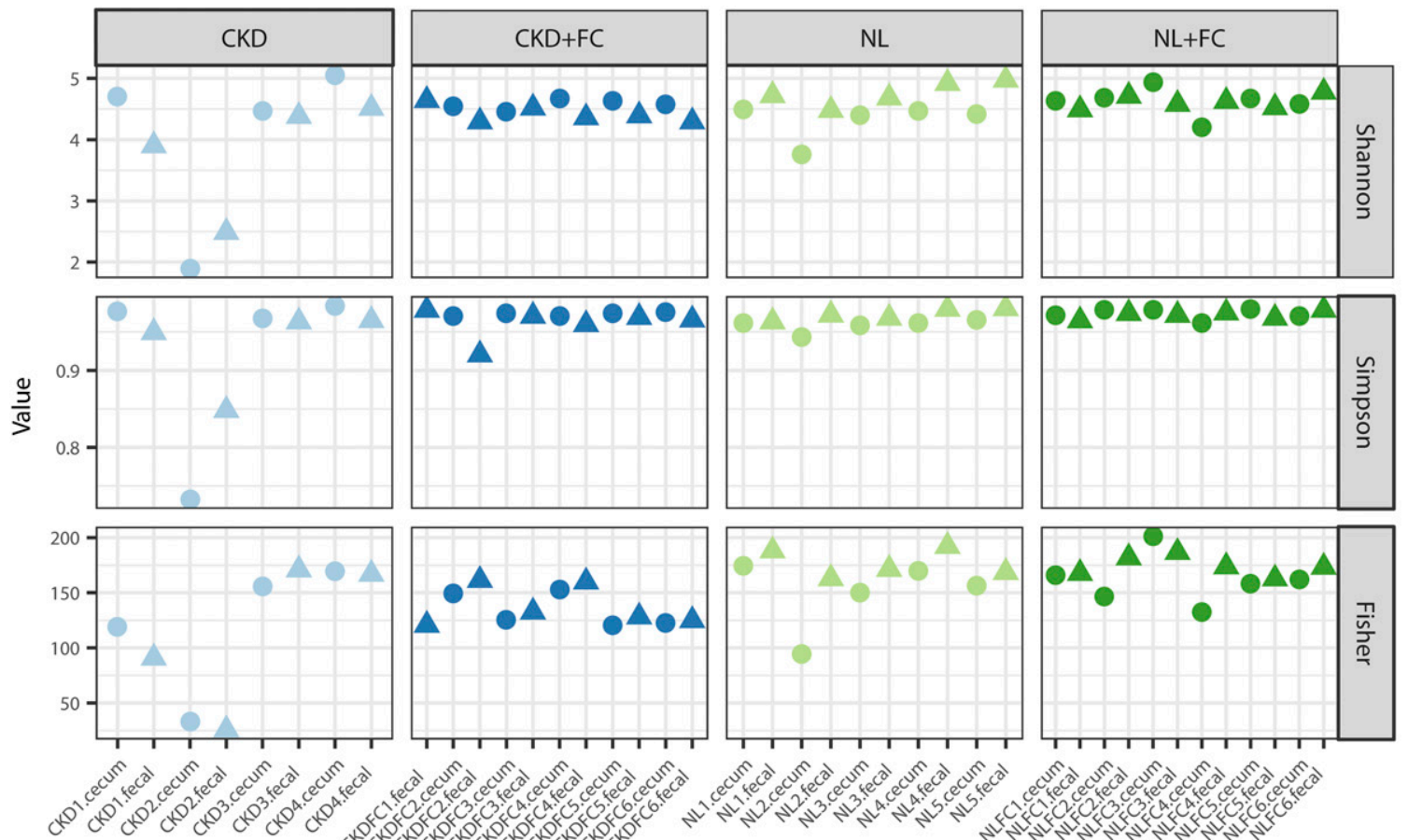

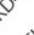
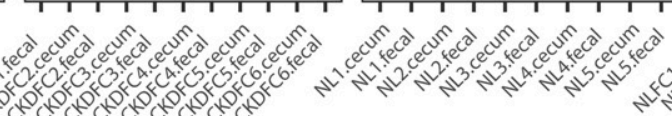

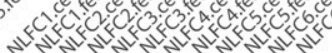

Sample

Fig. 3. $\alpha$-diversity metrics of rat fecal and cecal samples from untreated and FC-treated normal controls and CKD rats. Three $\alpha$-diversity metrics are shown: Shannon (top), Simpson (middle), and Fisher (bottom).

variation in microbial community composition. The control versus CKD groupings explained $9 \%(P<0.01)$ of the variance in the samples (Supplemental Table 1), whereas ferric citrate treatment explained about $11 \%$ of the variance $(P<0.01)$. Samples from the colonic feces or cecum had similar composition (Fig. 1) and clustered near one another (Fig. 4), suggesting that fecal samples were largely representative of cecal samples. However, the PERMANOVA result shows that there was a significant difference between the fecal and cecal samples, with a small effect size, explaining less than $5 \%$ of the variance (Supplemental Table 1).

\section{Discussion}

Our study supports prior reports (Aronov et al., 2011; Vaziri et al., 2013a; Wong et al., 2014) showing that the gut microbiome is markedly altered in CKD. CKD rats had lower and more variable gut microbial diversity. Both treated and untreated CKD rat microbial communities contained higher levels of Bacteroidetes and lower levels of Firmicutes, in contrast to normal animals that have more Firmicutes. Ferric citrate treatment in CKD rats increased diversity almost to the levels observed in normal animals and also brought the microbial community compositions across samples closer together. Thus, the "Anna Karenina hypothesis" may apply to the uremic microbiome, in which "all happy families look alike; each unhappy family is unhappy in its own way" (Zaneveld et al., 2017). Ferric citrate treatment also increased levels of tryptophanase-possessing families that are associated with production of indole and $p$-cresyl uremic toxins (Verrucomicrobia, Clostridiaceae, and Enterobacteriaceae); however, measured plasma levels of indoxyl sulfate and $p$-cresyl sulfate were not significantly different with ferric citrate treatment. The gut microbial changes were associated with improved kidney function (increased creatinine clearance, lower plasma creatinine) and decreased hypertension in the ferric citrate-treated CKD animals.

In an earlier study in hemodialysis patients, phylogenic microarray analysis of microbial DNA demonstrated highly significant differences in the abundance of more than 200 bacterial OTUs belonging to 23 bacterial families compared with healthy controls (Vaziri et al., 2013a). The OTUs that were markedly increased included the Cellulomonadaceae, Clostridiaceae, Enterobacteriaceae, Moraxellaceae, Pseudomonadaceae, and Verrucomicrobiaceae families (Vaziri et al., 2013a). The Clostridiaceae, Enterobacteriaceae, and Verrucomicrobiaceae are of particular interest because these microbes

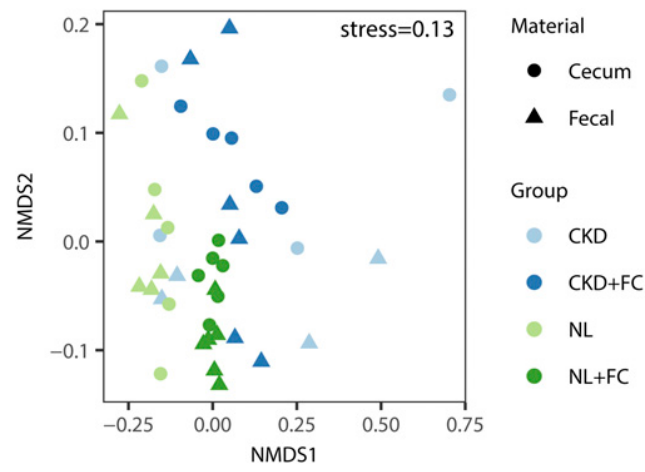

Fig. 4. An NMDS ordination plot of Bray-Curtis distances from $16 \mathrm{~S}$ ribosomal RNA gene sequences is shown for each sample, represented by treatment group and sample type. 
A

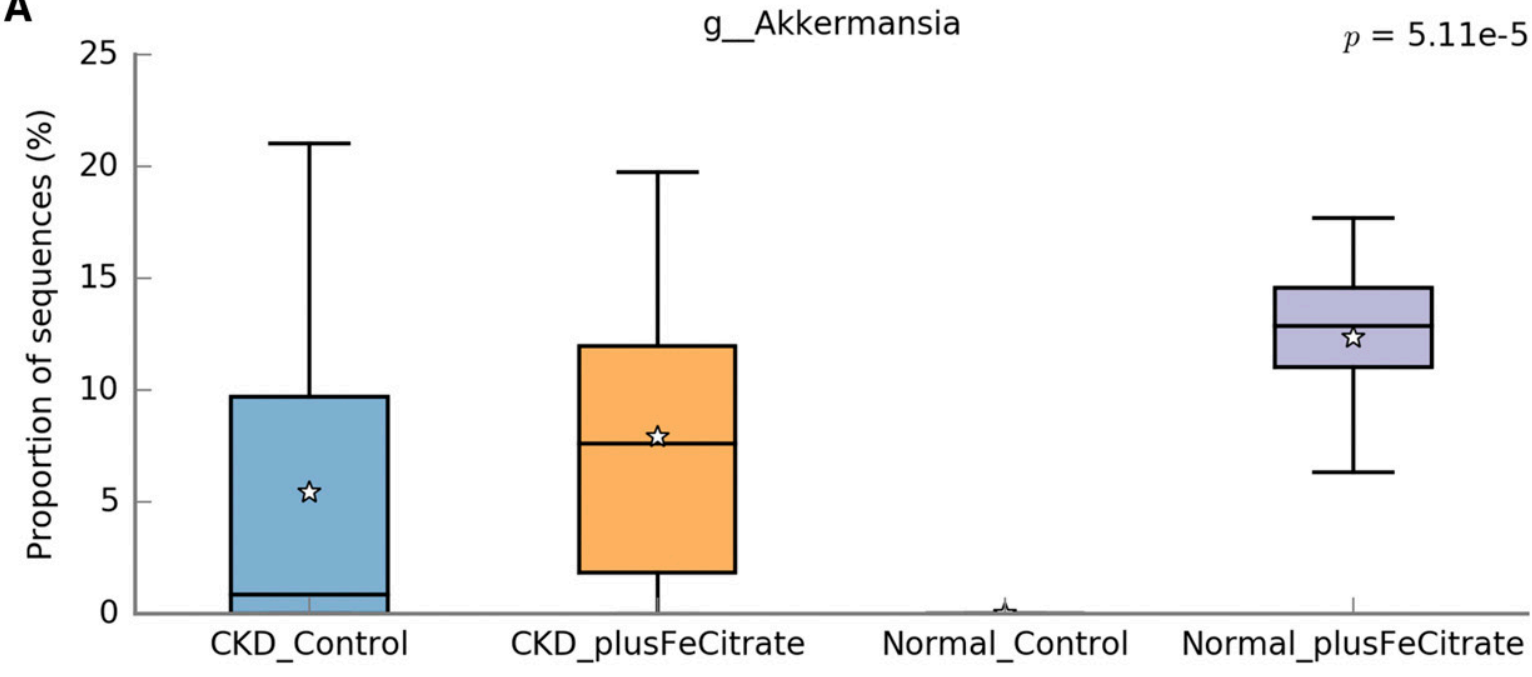

B

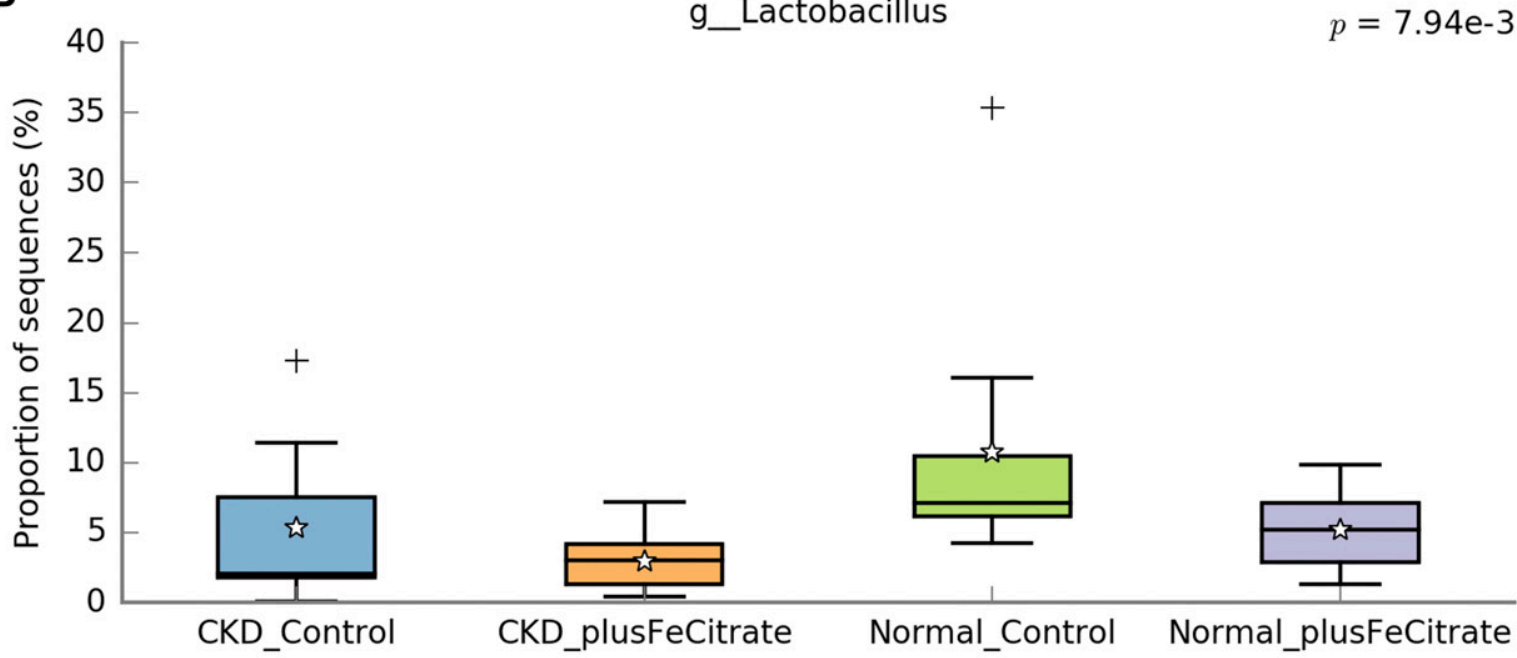

g_Akkermansia

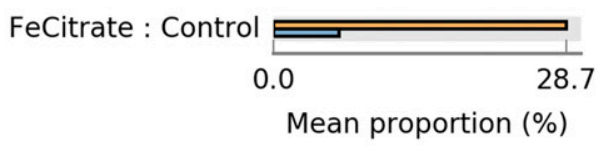

Mean proportion (\%)
$95 \%$ confidence intervals

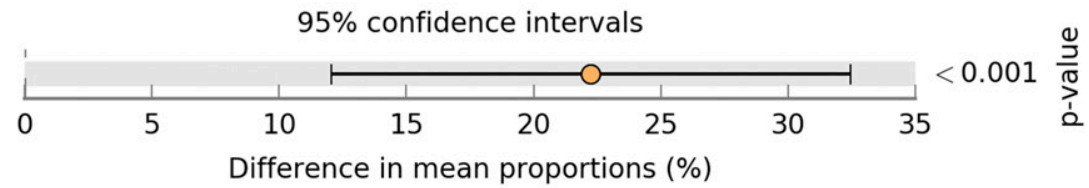

Difference in mean proportions (\%)

g_Lactobacillus
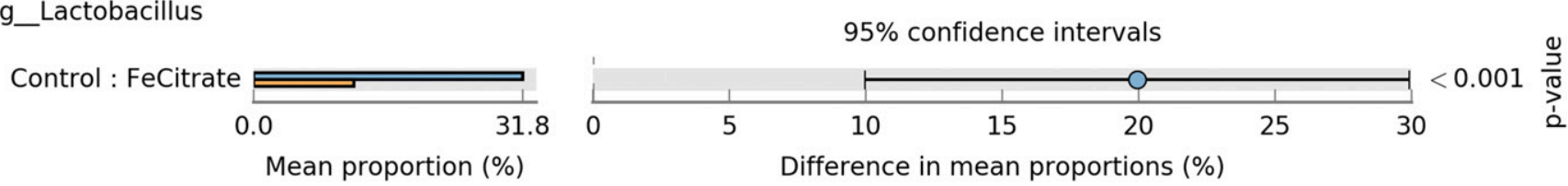

Fig. 5. Abundances of bacterial species amplicons that differed across the treatment groups. (A) Akkermansia spp. (B) Lactobacillus spp. Significance testing was done with the Tukey-Kramer post hoc test in STAMP software (see the Materials and Methods). Boxes indicate the inter-quartile range (IQR, 75th to 25th of the data). The median value is shown as a line within the box and the mean value as a star. Whiskers extend to the most extreme value within $1.5 * \mathrm{IQR}$. Outliers are shown as crosses $(+)$. 
possess indole- and $p$-cresyl-forming enzymes (i.e., tryptophanasepossessing families) (Wong et al., 2014) and generate gutderived uremic toxins such as indoxyl sulfate and $p$-cresyl sulfate that translocate back into the bloodstream and contribute to systemic inflammation (Mafra et al., 2014; Rossi et al., 2014; Lau et al., 2015).

In our animal study, ferric citrate therapy increased Clostridiaceae, Enterobacteriaceae, and Verrucomicrobiaceae in the stool from CKD animals; however, plasma levels of the tryptophan-derived uremic toxins indoxyl sulfate and $p$-cresyl sulfate were not significantly increased. Ferric citrate therapy was associated with improved kidney function, suggesting that a potentially deleterious increase in the production of gut-derived uremic toxins was offset by improved urinary clearance. Another pathway of interest may have been the increased abundance of $A$. muciniphila (Verrucomicrobiaceae). A. muciniphila is a mucin-consuming bacterium that may have an important role in maintaining the integrity of the intestinal mucosal barrier and has antiinflammatory properties. This species has been suggested as a biomarker of a healthy gut status (Fujio-Vejar et al., 2017) and may be induced by a low-fiber diet; however, it does not occur outside of the Western world (Desai et al., 2016; Cani and de Vos, 2017; Ottman et al., 2017; Smits et al., 2017). The increase in Akkermensia, akin to a reduction in dietary fiber, is interesting and raises further questions about whether ferric citrate influences gut mucins. Akkermansia metabolizes mucin to acetate and propionate, short-chain fatty acids that are nutrients for the host's enterocytes (Derrien et al., 2004, 2011), and creates a positive feedback loop that stimulates mucin secretion (Derrien et al., 2010). A. muciniphila has been shown to decrease endotoxemia and regulate adipose tissue metabolism and glucose homeostasis (Everard et al., 2013; Shin et al., 2014; Anhê et al., 2015).

Patients with end-stage kidney disease show decreased numbers of gut bacteria such as Lactobacillaceae and Prevotellaceae that are able to produce the short-chain fatty acid butyrate, also an important nutrient source for host enterocytes (Vaziri et al., 2013a; Wong et al., 2014). It was recently proposed that the use of oral iron supplements might further contribute to gut microbiome alterations (Kortman et al., 2017), extrapolating from studies in African children where supplemental iron decreased the abundances of bacteria considered to be beneficial, such as Bifidobacteriaceae and Lactobacillaceae (Jaeggi et al., 2015), and increased gut permeability (Nchito et al., 2006). In an in vitro model of the human colon, where intestinal epithelial Caco-2 cells were inoculated with human microbiota, incubation with ferrous sulfate or ferric citrate altered the microbiome population and also decreased levels of Bifidobacteriaceae and Lactobacillaceae (Kortman et al., 2016). The investigators noted cytotoxicity to Caco-2 cells with effluent from iron treatment conditions that contained microbe-derived metabolites (Kortman et al., 2016). Our study confirmed a decrease in Lactobacillus counts in both normal and CKD rats treated with ferric citrate; however, this did not translate to worse kidney function or blood pressure outcomes. It should be noted that unlike the soluble ferrous sulfate and ferric citrate compounds used in the above studies, the ferric citrate employed in our study is an extremely large and insoluble complex, which is used as a phosphate binder.
Ferric citrate has other beneficial effects that may have overwhelmed any systemic impact from the altered gut microbiome. As a phosphate binder, ferric citrate ameliorates hyperphosphatemia, secondary hyperparathyroidism, and vascular calcification (Yokoyama et al., 2012, 2014; Iida et al., 2013; Block et al., 2015; Lee et al., 2015; Lewis et al., 2015). Furthermore, ferric citrate improves iron deficiency anemia (Fishbane et al., 2017), as evidenced by the increased hemoglobin levels in the treated CKD rats.

Our study is not able to define to what extent the microbiome alterations contributed to overall systemic benefits, as opposed to direct effects of ferric citrate itself. Future investigations with germ-free rodents would be one way to separate the impact of ferric citrate on the host alone, independent of the microbiota. Furthermore, there is the emerging concept of the microgenderome, whereby sex differences in the microbiome may influence systemic outcomes (Flak et al., 2013; Markle et al., 2013; Elderman et al., 2018); the current ferric citrate investigation will need to be replicated in female CKD rats to delineate sex differences in measured outcomes.

In summary, our study suggests that CKD is associated with lower and more variable gut microbial diversity. Ferric citrate therapy decreased hyperphosphatemia, improved anemia, and improved gut microbial diversity almost to the levels observed in normal animals. Lactobacillaceae were further decreased with ferric citrate therapy, whereas Verrucomicrobiaceae increased. Of particular interest within the Verrucomicrobiaceae is A. muciniphila, which has anti-inflammatory properties and promotes integrity of the intestinal barrier. The gut-derived uremic toxins indoxyl sulfate and $p$-cresyl sulfate were not significantly altered with ferric citrate therapy. The CKD rats treated with ferric citrate had less hypertension and better kidney function, as assessed by plasma creatinine and urinary creatinine clearance. Overall, our findings support a beneficial impact of oral ferric citrate in $\mathrm{CKD}$ in terms of promoting gut microbial diversity and improved kidney function.

\section{Authorship Contributions}

Participated in research design: Lau, Vaziri, Whiteson.

Conducted experiments: Lau, Nunes, Comeau, Langille, England, Khazaeli, Suematsu, Phan.

Contributed new reagents or analytic tools: Langille, Whiteson. Performed data analysis: Lau, Vaziri, Nunes, England, Whiteson Wrote or contributed to the writing of the manuscript: Lau, Vaziri, Nunes, Comeau, Langille, England, Whiteson.

\section{References}

Alexeev EE, He X, Slupsky CM, and Lönnerdal B (2017) Effects of iron supplementation on growth, gut microbiota, metabolomics and cognitive development of rat pups. PLoS One 12:e0179713.

Anhê FF, Roy D, Pilon G, Dudonné S, Matamoros S, Varin TV, Garofalo C, Moine Q, Desjardins Y, Levy E, et al. (2015) A polyphenol-rich cranberry extract protects from diet-induced obesity, insulin resistance and intestinal inflammation in association with increased Akkermansia spp. population in the gut microbiota of mice. Gut 64:872-883.

Aronov PA, Luo FJ, Plummer NS, Quan Z, Holmes S, Hostetter TH, and Meyer TW (2011) Colonic contribution to uremic solutes. J Am Soc Nephrol 22:1769-1776.

Benoni G, Cuzzolin L, Zambreri D, Donini M, Del Soldato P, and Caramazza I (1993) Gastrointestinal effects of single and repeated doses of ferrous sulphate in rats. Pharmacol Res 27:73-80.

Block GA, Fishbane S, Rodriguez M, Smits G, Shemesh S, Pergola PE, Wolf M, and Chertow GM (2015) A 12-week, double-blind, placebo-controlled trial of ferric citrate for the treatment of iron deficiency anemia and reduction of serum phosphate in patients with CKD stages 3-5. Am J Kidney Dis 65:728-736.

Cachofeiro V, Goicochea M, de Vinuesa SG, Oubiña P, Lahera V, and Luño J (2008) Oxidative stress and inflammation, a link between chronic kidney disease and cardiovascular disease. Kidney Int Suppl 111:S4-S9.

Cani PD and de Vos WM (2017) Next-generation beneficial microbes: the case of Akkermansia muciniphila. Front Microbiol 8:1765. 
Comeau AM, Douglas GM, and Langille MG (2017) Microbiome Helper: a custom and streamlined workflow for microbiome research. mSystems 2:e00127-16.

Derrien M, Van Baarlen P, Hooiveld G, Norin E, Müller M, and de Vos WM (2011) Modulation of mucosal immune response, tolerance, and proliferation in mice colonized by the mucin-degrader Akkermansia muciniphila. Front Microbiol 2:166.

Derrien M, van Passel MW, van de Bovenkamp JH, Schipper RG, de Vos WM, and Dekker J (2010) Mucin-bacterial interactions in the human oral cavity and digestive tract. Gut Microbes 1:254-268.

Derrien M, Vaughan EE, Plugge CM, and de Vos WM (2004) Akkermansia muciniphila gen. nov., sp. nov., a human intestinal mucin-degrading bacterium. Int J Syst Evol Microbiol 54:1469-1476.

Desai MS, Seekatz AM, Koropatkin NM, Kamada N, Hickey CA, Wolter M, Pudlo NA, Kitamoto S, Terrapon N, Muller A, et al. (2016) A dietary fiber-deprived gut microbiota degrades the colonic mucus barrier and enhances pathogen susceptibility, Cell 167:1339-1353.e21.

Elderman M, Hugenholtz F, Belzer C, Boekschoten M, van Beek A, de Haan B, Savelkoul H, de Vos P, and Faas M (2018) Sex and strain dependent differences in mucosal immunology and microbiota composition in mice. Biol Sex Differ 9:26.

Everard A, Belzer C, Geurts L, Ouwerkerk JP, Druart C, Bindels LB, Guiot Y, Derrien M, Muccioli GG, Delzenne NM, et al. (2013) Cross-talk between Akkermansia muciniphila and intestinal epithelium controls diet-induced obesity. Proc Natl Acad Sci USA 110:9066-9071.

Fishbane S, Block GA, Loram L, Neylan J, Pergola PE, Uhlig K, and Chertow GM (2017) Effects of ferric citrate in patients with nondialysis-dependent CKD and iron deficiency anemia. J Am Soc Nephrol 28:1851-1858.

Flak MB, Neves JF, and Blumberg RS (2013) Immunology. Welcome to the microgenderome. Science 339:1044-1045.

Fujio-Vejar S, Vasquez Y, Morales P, Magne F, Vera-Wolf P, Ugalde JA, Navarrete P, and Gotteland M (2017) The gut microbiota of healthy Chilean subjects reveals a high abundance of the phylum Verrucomicrobia. Front Microbiol 8:1221.

Heine GH, Ortiz A, Massy ZA, Lindholm B, Wiecek A, Martínez-Castelao A, Covic A, Goldsmith D, Süleymanlar G, London GM, et al.; European Renal and Cardiovascular Medicine (EURECA-m) working group of the European Renal Association-European Dialysis and Transplant Association (ERA-EDTA) (2012) Monocyte subpopulations and cardiovascular risk in chronic kidney disease. Nat Rev Nephrol 8:362-369.

Himmelfarb J, Stenvinkel P, Ikizler TA, and Hakim RM (2002) The elephant in uremia: oxidant stress as a unifying concept of cardiovascular disease in uremia. Kidney Int 62:1524-1538.

Holden VI and Bachman MA (2015) Diverging roles of bacterial siderophores during infection. Metallomics 7:986-995.

Iida A, Kemmochi Y, Kakimoto K, Tanimoto M, Mimura T, Shinozaki Y, Uemura A, Matsuo A, Matsushita M, and Miyamoto K (2013) Ferric citrate hydrate, a new phosphate binder, prevents the complications of secondary hyperparathyroidism and vascular calcification. Am J Nephrol 37:346-358.

Jaeggi T, Kortman GA, Moretti D, Chassard C, Holding P, Dostal A, Boekhorst J, Timmerman HM, Swinkels DW, Tjalsma H, et al. (2015) Iron fortification adversely affects the gut microbiome, increases pathogen abundance and induces intestinal inflammation in Kenyan infants. Gut 64:731-742.

Kanemitsu Y, Asaji K, Matsumoto Y, Tsukamoto H, Saigusa D, Mukawa C, Tachikawa T, Abe T, and Tomioka Y (2017) Simultaneous quantitative analysis of uremic toxins by LC-MS/MS with a reversed-phase/cation-exchange/anionexchange tri-modal mixed-mode column. J Chromatogr B Analyt Technol Biomed Life Sci 1068-1069:1-8.

Kato S, Chmielewski M, Honda H, Pecoits-Filho R, Matsuo S, Yuzawa Y, Tranaeus A, Stenvinkel P, and Lindholm B (2008) Aspects of immune dysfunction in end-stage renal disease. Clin J Am Soc Nephrol 3:1526-1533.

Kieffer DA, Piccolo BD, Vaziri ND, Liu S, Lau WL, Khazaeli M, Nazertehrani S, Moore ME, Marco ML, Martin RJ, et al. (2016) Resistant starch alters gut microbiome and metabolomic profiles concurrent with amelioration of chronic kidney disease in rats. Am J Physiol Renal Physiol 310:F857-F871.

Kortman GA, Dutilh BE, Maathuis AJ, Engelke UF, Boekhorst J, Keegan KP, Nielsen FG, Betley J, Weir JC, Kingsbury Z, et al. (2016) Microbial metabolism shifts towards an adverse profile with supplementary iron in the TIM-2 in vitro model of the human colon. Front Microbiol 6:1481.

Kortman GAM, Reijnders D, and Swinkels DW (2017) Oral iron supplementation potential implications for the gut microbiome and metabolome in patients with CKD. Hemodial Int 21 (Suppl 1):S28-S36.

Lau WL, Kalantar-Zadeh K, and Vaziri ND (2015) The gut as a source of inflammation in chronic kidney disease. Nephron 130:92-98.

Lee CT, Wu IW, Chiang SS, Peng YS, Shu KH, Wu MJ, and Wu MS (2015) Effect of oral ferric citrate on serum phosphorus in hemodialysis patients: multicenter, randomized, double-blind, placebo-controlled study. J Nephrol 28:105-113.

Lewis JB, Sika M, Koury MJ, Chuang P, Schulman G, Smith MT, Whittier FC, Linfert DR, Galphin CM, Athreya BP, et al.; Collaborative Study Group (2015) Ferric citrate controls phosphorus and delivers iron in patients on dialysis. J Am Soc Nephrol 26:493-503.

Liabeuf S, Barreto DV, Barreto FC, Meert N, Glorieux G, Schepers E, Temmar M, Choukroun G, Vanholder R, and Massy ZA; European Uraemic Toxin Work Group (EUTox) (2010) Free p-cresylsulphate is a predictor of mortality in patients at different stages of chronic kidney disease. Nephrol Dial Transplant 25: $1183-1191$

Mafra D, Lobo JC, Barros AF, Koppe L, Vaziri ND, and Fouque D (2014) Role of altered intestinal microbiota in systemic inflammation and cardiovascular disease in chronic kidney disease. Future Microbiol 9:399-410.

Magnusson M, Magnusson KE, Sundqvist T, and Denneberg T (1990) Increased intestinal permeability to differently sized polyethylene glycols in uremic rats: effects of low- and high-protein diets. Nephron 56:306-311.
Magnusson M, Magnusson KE, Sundqvist T, and Denneberg T (1991) Impaired intestinal barrier function measured by differently sized polyethylene glycols in patients with chronic renal failure. Gut 32:754-759.

Markle JG, Frank DN, Mortin-Toth S, Robertson CE, Feazel LM, Rolle-Kampczyk U, von Bergen M, McCoy KD, Macpherson AJ, and Danska JS (2013) Sex differences in the gut microbiome drive hormone-dependent regulation of autoimmunity. Science 339:1084-1088.

McIntyre CW, Harrison LE, Eldehni MT, Jefferies HJ, Szeto CC, John SG, Sigrist MK, Burton JO, Hothi D, Korsheed S, et al. (2011) Circulating endotoxemia: a novel factor in systemic inflammation and cardiovascular disease in chronic kidney disease. Clin J Am Soc Nephrol 6:133-141.

Nchito M, Friis H, Michaelsen KF, Mubila L, and Olsen A (2006) Iron supplementation increases small intestine permeability in primary schoolchildren in Lusaka, Zambia. Trans $R$ Soc Trop Med Hyg 100:791-794.

Oksanen J, Kindt R, Legendre P, O'Hara B, Stevens MHH, Oksanen MJ, and Suggests M (2007) The vegan package. Community Ecology Package 10: 631-637.

Ormerod KL, Wood DL, Lachner N, Gellatly SL, Daly JN, Parsons JD, Dal'Molin CG, Palfreyman RW, Nielsen LK, Cooper MA, et al. (2016) Genomic characterization of the uncultured Bacteroidales family S24-7 inhabiting the guts of homeothermic animals. Microbiome 4:36.

Ottman N, Geerlings SY, Aalvink S, de Vos WM, and Belzer C (2017) Action and function of Akkermansia muciniphila in microbiome ecology, health and disease. Best Pract Res Clin Gastroenterol 31:637-642.

Parks DH, Tyson GW, Hugenholtz P, and Beiko RG (2014) STAMP: statistical analysis of taxonomic and functional profiles. Bioinformatics 30:3123-3124.

Rossi M, Campbell KL, Johnson DW, Stanton T, Vesey DA, Coombes JS, Weston KS, Hawley CM, McWhinney BC, Ungerer JP, et al. (2014) Protein-bound uremic toxins, inflammation and oxidative stress: a cross-sectional study in stage 3-4 chronic kidney disease. Arch Med Res 45:309-317.

Shin NR, Lee JC, Lee HY, Kim MS, Whon TW, Lee MS, and Bae JW (2014) An increase in the Akkermansia spp. population induced by metformin treatment improves glucose homeostasis in diet-induced obese mice. Gut 63:727-735.

Shu C, Chen X, Xia T, Zhang F, Gao S, and Chen W (2016) LC-MS/MS method for simultaneous determination of serum p-cresyl sulfate and indoxyl sulfate in patients undergoing peritoneal dialysis. Biomed Chromatogr 30:1782-1788.

Sirich TL, Plummer NS, Gardner CD, Hostetter TH, and Meyer TW (2014) Effect of increasing dietary fiber on plasma levels of colon-derived solutes in hemodialysis patients. Clin J Am Soc Nephrol 9:1603-1610.

Smits SA, Leach J, Sonnenburg ED, Gonzalez CG, Lichtman JS, Reid G, Knight R, Manjurano A, Changalucha J, Elias JE, et al. (2017) Seasonal cycling in the gut microbiome of the Hadza hunter-gatherers of Tanzania. Science 357: 802-806.

Stubbs JR, House JA, Ocque AJ, Zhang S, Johnson C, Kimber C, Schmidt K, Gupta A, Wetmore JB, Nolin TD, et al. (2016) Serum trimethylamine-N-oxide is elevated in CKD and correlates with coronary atherosclerosis burden. J Am Soc Nephrol 27: $305-313$

Szeto CC, Kwan BC, Chow KM, Lai KB, Chung KY, Leung CB, and Li PK (2008) Endotoxemia is related to systemic inflammation and atherosclerosis in peritoneal dialysis patients. Clin J Am Soc Nephrol 3:431-436.

Tang WH, Wang Z, Kennedy DJ, Wu Y, Buffa JA, Agatisa-Boyle B, Li XS, Levison BS, and Hazen SL (2015) Gut microbiota-dependent trimethylamine N-oxide (TMAO) pathway contributes to both development of renal insufficiency and mortality risk in chronic kidney disease. Circ Res 116:448-455

Tompkins GR, O'Dell NL, Bryson IT, and Pennington CB (2001) The effects of dietary ferric iron and iron deprivation on the bacterial composition of the mouse intestine. Curr Microbiol 43:38-42.

Vaziri ND (2004) Oxidative stress in uremia: nature, mechanisms, and potential consequences. Semin Nephrol 24:469-473.

Vaziri ND, Bai Y, Ni Z, Quiroz Y, Pandian R, and Rodriguez-Iturbe B (2007) Intrarenal angiotensin II/AT1 receptor, oxidative stress, inflammation, and progressive injury in renal mass reduction. J Pharmacol Exp Ther 323:85-93.

Vaziri ND, Ni Z, Oveisi F, Liang K, and Pandian R (2002) Enhanced nitric oxide inactivation and protein nitration by reactive oxygen species in renal insufficiency. Hypertension 39:135-141.

Vaziri ND, Wong J, Pahl M, Piceno YM, Yuan J, DeSantis TZ, Ni Z, Nguyen TH, and Andersen GL (2013a) Chronic kidney disease alters intestinal microbial flora. Kidney Int 83:308-315.

Vaziri ND, Yuan J, Nazertehrani S, Ni Z, and Liu S (2013b) Chronic kidney disease causes disruption of gastric and small intestinal epithelial tight junction. Am $J$ Nephrol 38:99-103.

Vaziri ND, Yuan J, and Norris K (2013c) Role of urea in intestinal barrier dysfunction and disruption of epithelial tight junction in chronic kidney disease. Am J Nephrol 37:1-6.

Vaziri ND, Yuan J, Rahimi A, Ni Z, Said H, and Subramanian VS (2012) Disintegration of colonic epithelial tight junction in uremia: a likely cause of CKDassociated inflammation. Nephrol Dial Transplant 27:2686-2693.

Vaziri ND, Zhao YY, and Pahl MV (2016) Altered intestinal microbial flora and impaired epithelial barrier structure and function in CKD: the nature, mechanisms, consequences and potential treatment. Nephrol Dial Transplant 31: $737-746$.

Wang F, Zhang P, Jiang H, and Cheng S (2012) Gut bacterial translocation contributes to microinflammation in experimental uremia. Dig Dis Sci 57:2856-2862.

Wilson BR, Bogdan AR, Miyazawa M, Hashimoto K, and Tsuji Y (2016) Siderophores in iron metabolism: from mechanism to therapy potential. Trends Mol Med 22: 1077-1090

Wong J, Piceno YM, DeSantis TZ, Pahl M, Andersen GL, and Vaziri ND (2014) Expansion of urease- and uricase-containing, indole- and p-cresol-forming and contraction of short-chain fatty acid-producing intestinal microbiota in ESRD. Am J Nephrol 39:230-237. 
Yokoyama K, Akiba T, Fukagawa M, Nakayama M, Sawada K, Kumagai Y, Chertow GM, and Hirakata H (2014) A randomized trial of JTT-751 versus sevelamer hydrochloride in patients on hemodialysis. Nephrol Dial Transplant 29:1053-1060.

Yokoyama K, Hirakata H, Akiba T, Sawada K, and Kumagai Y (2012) Effect of oral JTT-751 (ferric citrate) on hyperphosphatemia in hemodialysis patients: results of a randomized, double-blind, placebo-controlled trial. Am J Nephrol 36:478-487.

Yoon JW, Pahl MV, and Vaziri ND (2007) Spontaneous leukocyte activation and oxygen-free radical generation in end-stage renal disease. Kidney Int $\mathbf{7 1}$ $167-172$.
Zaneveld JR, McMinds R, and Vega Thurber R (2017) Stress and stability: applying the Anna Karenina principle to animal microbiomes. Nat Microbiol 2:17121.

Address correspondence to: Dr. Katrine Whiteson, Department of Molecular Biology and Biochemistry, 3236 (office), 3315 (laboratory) McGaugh Hall, University of California, Irvine, CA 92697. E-mail: katrine@uci.edu; or Dr. Wei Ling Lau, Division of Nephrology and Hypertension, Department of Medicine, University of California, 333 City Blvd. West, Suite 400, Orange, CA 92868. E-mail: wllau@uci.edu 